\title{
MALNUTRITION FOLLOWING STROKE IN A REHABILITATION UNIT: FREQUENCY AND ASSOCIATED RISK FACTORS
}

\author{
Ayşe A. Küçükdeveci, Diana Rüstemova Bayraktar, Atilla H. Elhan*, Şehim Kutlay \\ Department of Physical Medicine and Rehabilitation, Ankara University, Faculty of Medicine, Ankara, Turkey \\ *Department of Biostatistics, Ankara University, Faculty of Medicine, Ankara, Turkey
}

Introduction: Prevalance of malnutrition following stroke is reported to vary widely between $6 \%$ and $62 \%$.

Purpose: The aim of this cross-sectional study was to investigate the frequency of malnutrition and its associated risk factors in stroke patients admitted for rehabilitation in a rehabilitation unit of a university hospital.

\section{Methods:}

Patients' nutritional status was determined by the amount of weight loss and body mass index (BMI):

- Patients were defined as severely undernourished: BMI $<18.5$ (or BMI $<20$ for patients age $\geq 65$ years) and/or $>5 \%$ unintentional weight loss in the past month and/or $>10 \%$ unintentional weight loss in the past 6 months.

- Patients age $\geq 65$ years were defined as moderately undernourished: BMI 20-22 and/or 5-10\% weight loss in the past 6 months.

- Patients age $<65$ years were defined as moderately undernourished: BMI 18.5-22 and/or 5-10\% unintentional weight loss in the past 6 months

Malnutrition risk was determined by Nutritional Risk Screening-2002 (NRS 2002).

All patients were questioned and evaluated regarding parameters which might be associated with malnutrition:

- Demographic: Age, sex, education, marital status

- Clinical: Time since stroke, previous history of pneumonia, dysphagia and pressure ulcers, comorbidities, smoking, alcohol use, stroke type, hemiplegic side, mid-upper arm circumference, triceps skinfold thickness, feeding method

- Biochemical: Albumin, prealbumin, transferrin, protein, 25(OH)Vitamin D, Vitamin B12, zinc

- Function: Functioning by Functional Indepence Measure=FIMTM, global disability level by Modified Rankin Scale.

\section{Results:}

71 stroke patients were included:

- Mean age was $60.2 \pm 13.8$ years, $43 \%$ were male, median time since stroke was 6.2 months (range: $0.3-130$ months)

$22.5 \%(n=16)$ of the patients were found to be malnourished, $7 \%$ severely and $15.5 \%$ moderately malnourished.

$29.6 \%$ of the patients were at the risk of malnutrition according to NRS 2002. All patients with malnutrition risk were malnourished. In addition 5 people without malnutrition were at risk according to NRS 2002.

In the malnourished group, time since stroke was shorter, motor functioning (motor FIM) and global disability levels (Modified Rankin scores) were worse $(\mathrm{p}<0.05)$.

Patients who had previous history of pneumonia or pressure sores, whose feeding method was Nasogastric/PEG and whose mid-upper arm circumference (MUAC) and serum prealbumin levels below the cut-off were more prevalant in the malnourished group $(p<0.05)$.

In univariate regression analysis, time since stroke ( $\leq 12$ months), educational level (university or higher), previous history of pnemonia or pressure sores, MUAC $(\leq 25.5 \mathrm{~cm})$, method of feeding (Nasogastric/PEG) and serum prealbumin $(\leq 0.18 \mathrm{~g} / \mathrm{L})$ were found to be significant risk factors.

Multiple regression analysis revealed that time since stroke $\leq 12$ months $(p=0.010)$, MUAC $\leq 25.5 \mathrm{~cm}(p=0.007)$ and serum prealbümin levels $\leq 0.18 \mathrm{~g} / \mathrm{L}(p=0.012)$ were independent risk factors which could predict malnutrition risk.

\section{Conclusion:}

- Frequency of malnutrition was $22.5 \%$ in a group of stroke patients, admitted for rehabilitation at acute, post-acute and chronic stages.

- Time since stroke less than 12 months, MUAC and serum prealbumin levels below the cut-off points were found as independent risk factors for malnutrition following stroke.

\section{References:}

Hertroijs D, et al. Rehabilitation patients: undernourished and obese? J Rehabil Med, 2012. 44(8): p. 696-701.

Meijers JM, et al. Malnutrition in Dutch health care: prevalence, prevention, treatment, and quality indicators. Nutrition, 2009;25(5): p.512-9.

Foley NC, et al. A review of the relationship between dysphagia and malnutrition following stroke. J Rehabil Med, 2009;41(9):707-13.

Mosselman MJ, et al. Malnutrition and risk of malnutrition in patients with stroke: prevalence during hospital stay. J Neurosci Nurs, 2013;45(4):194-204. 